\title{
Mechanical AGN feedback, from very powerful to moderately strong high-redshift radio galaxies
}

\author{
Cédric Collet ${ }^{* \dagger}$ \\ Institut d'Astrophysique Spatiale, Université Paris-Sud, 91405 Orsay, France \\ E-mail: cedric.collet@ias.u-psud.fr
}

\section{Nicole Nesvadba}

Institut d'Astrophysique Spatiale, CNRS, Université Paris-Sud, 91405 Orsay, France

E-mail: nicole.nesvadba@ias.u-psud.fr

\begin{abstract}
Galaxy-wide winds of ionized gas have shown that AGN can terminate star formation in the most powerful radio galaxies at $\mathrm{z} \sim 2$, but these are rare objects and 1000 times brighter in the radio than typical dusty high-z starbursts. We have observed 50 high-z radio galaxies with the VLT/SINFONI IFU to explore the systematics of AGN-driven winds from the most powerful radio sources to sources with moderate radio power. We report on the latest results of this ongoing study.
\end{abstract}

Nuclei of Seyfert Galaxies and QSOs - Central Engine and Conditions of Star Formation, November 6-8, 2012

Max-Planck-Insitut für Radioastronomie (MPIfR), Bonn, Germany

\footnotetext{
*Speaker.

${ }^{\dagger}$ Based on ESO observations
} 


\section{Motivations}

Feedback is more and more considered to be of crucial importance in our understanding of galaxy evolution (e.g., Silk \& Rees 1998; Croton et al. 2006). The consensus for a scenario in which massive galaxies experience a strong starburst, triggered by mergers and cooling flows and soon thereafter terminated by the AGN, which can efficiently heat the gas, is supported by observations over a large wavelength range. At low redshift, hot X-ray cavities are observed in galaxy clusters and seem to be inflated by radio jets, thus suppressing gas cooling (e.g., Birzan et al. 2004; Wise et al. 2007). At higher redshift, rest-frame visible emission lines of ionized gas, observed with integral field spectrographs, tend to show that radio jets are able to couple with the surounding gas and drive it out of the potential well of the galaxy (e.g., Nesvadba et al. 2006, 2008). Observations of some high-redshift sub-millimeter galaxies show that these much more common objects are also subject to some kind of feedback, plausibly radiatively driven by the AGN and/or supernovae from a starburst (e.g., Alexander et al. 2010; Harrison et al. 2012).

It is now well established that the key streps in the evolution of massive galaxies were already completed at high-redshift $(\mathrm{z} \gtrsim 2)$. Their star formation was maximum and has declined then (e.g., Karim et al. 2011), the luminosity function of quasars peaks at this period (e.g., Croom et al. 2009) and a rapidly evolving population of powerful radio galaxies appears at that time with co-moving number densities orders of magnitude higher than those observed in the local Universe (e.g., Willott et al. 2001; Gendre et al. 2010).

All these are possible sources of feedback, making it important to investigate which one dominates in what type of object and at what stage of evolution towards "old, red and dead" galaxies.
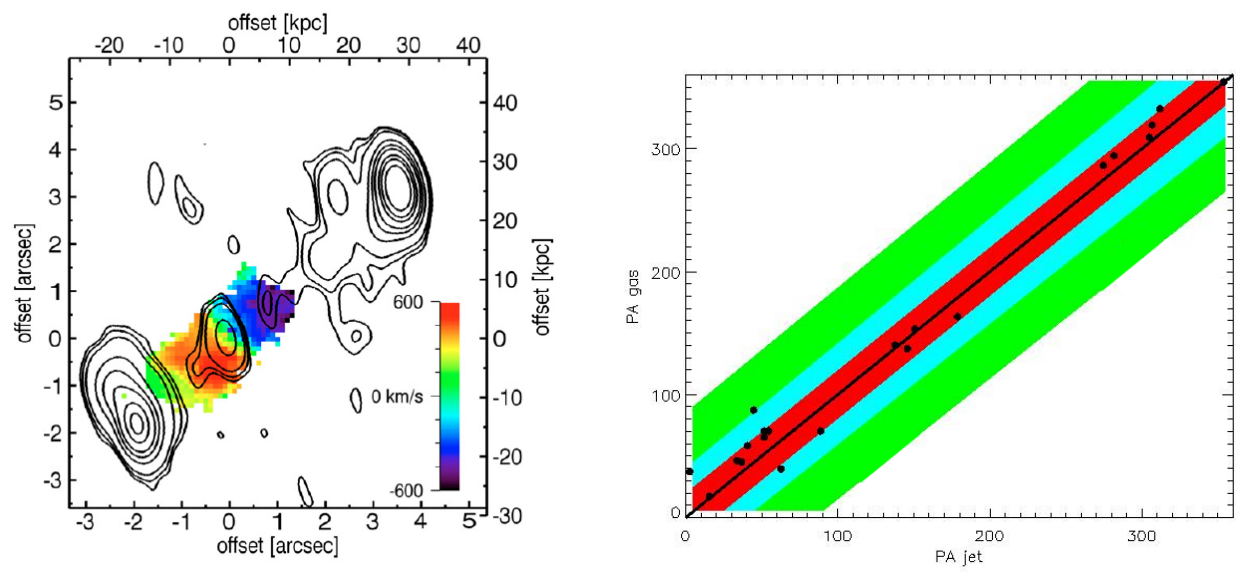

Figure 1: Left: The case study of MRC0406-244 (Nesvadba et al. 2008), in which radio jets are driving dramatic outflows. Right: Position angle of the ionized gas versus position angle of the radio jets: it appears that the ionized gas axis is always well-aligned with the radio jets axis, a strong argument for mechanical AGN feedback. 


\section{Results for powerful HzRG}

From observations of powerful high-redshft radio galaxies (HzRG) with $\mathscr{L}_{1.4 \mathrm{GHz}} \geq 10^{28} \mathrm{~W}$ $\mathrm{Hz}^{-1}$, mechanical feedback through radio jets seems to be the main driver of outflows in these kind of "monsters" (Nesvadba et al. 2006, 2008). This is well illustrated by the case of MRC0406-244, reproduced on Fig. 1: Large quantities of ionized gas $\left(M_{\text {ion gas }} \sim 10^{9-10} M_{\odot}\right)$ are outflowing with large velocities $\left(\mathrm{v} \sim 1000-1500 \mathrm{~km} \mathrm{~s}^{-1}\right)$ and large velocity dispersions $\left(\sigma \sim 1000 \mathrm{~km} \mathrm{~s}^{-1}\right)$ from the galaxy, driven by radio jets.

This example is typical of what is observed for the most powerful radio galaxies observed with SINFONI, where the gas is always well-aligned with the jet, as shown by Fig. 1.

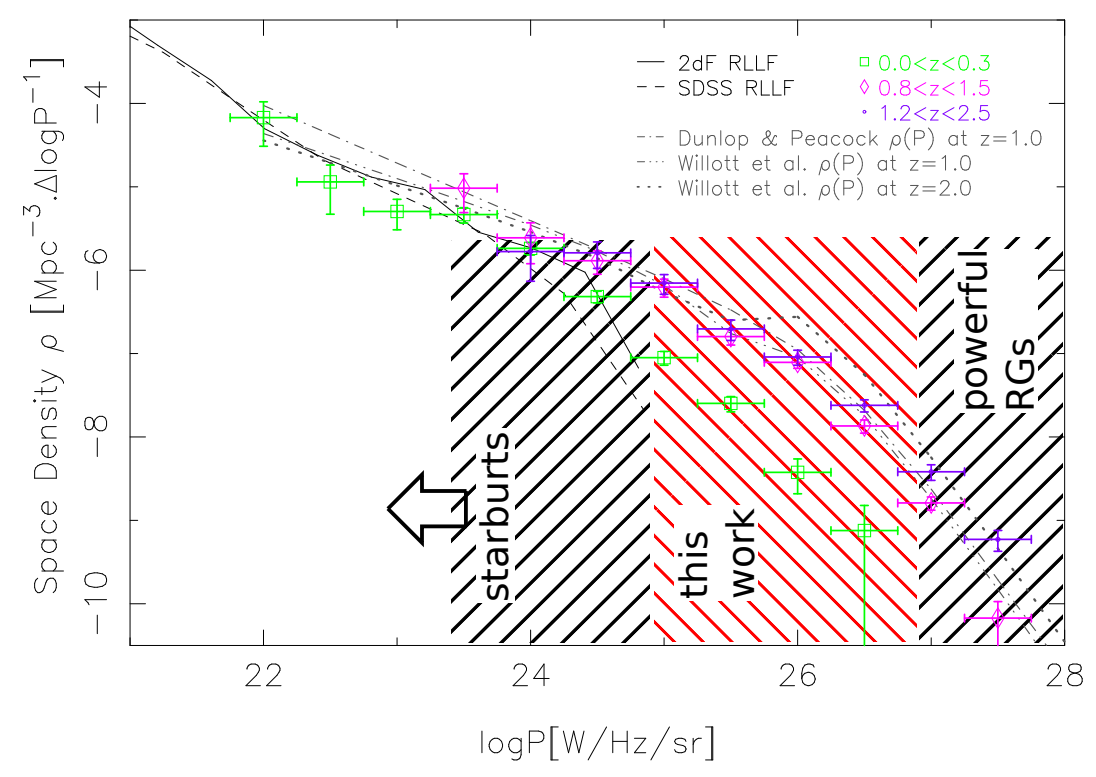

Figure 2: Radio luminosity function (from Gendre et al. 2010). It illustrates that the number density of radio-galaxies dramatically increases when radio power decreases, by a factor 100-1000 between the most powerful HzRG (already studied by Nesvadba et al., 2006, 2008) and those studied here. It also illustrates that radio galaxies were much more common (by 1-2 oders of magnitude) in the high-redshift Universe than locally.

However, at lower radio powers $\left(\mathscr{L}_{1.4 \mathrm{GHz}} \lesssim 10^{24-25} \mathrm{~W} \mathrm{~Hz}^{-1}\right)$, it is not clear whether radiative feedback dominates, as possibly illustrated by Alexander et al. (2010); or if radio jets continue to be the main driver, as postulated by Nesvadba et al. (2011). However, a large gap between those two examples still exists, all the more since a non-negligeable fraction of high redshift $(\mathrm{z} \sim 2)$ ULIRGs have radio jets produced by an AGN (e.g., Sajina et al. 2008). In this study, we try to fill this gap by studying a sample of 9 moderately strong radio galaxies, having $\mathscr{L}_{1.4 \mathrm{GHz}} \sim 10^{27} \mathrm{~W}$ $\mathrm{Hz}^{-1}$. This kind of HzRG is $100-1000$ times more common than those studied previously (see 
Fig. 2) and could therefore shed light on the prevalence of mechanical AGN feedback in more classical HzRGs.

\section{Our study}

We observed 9 galaxies with intermediate radio power $\left(\mathscr{L}_{1.4 \mathrm{GHz}} \sim 10^{27} \mathrm{~W} \mathrm{~Hz}^{-1}\right)$ with the near-infrared imaging spectrograph SINFONI (Eisenhauer et al. 2003 ; Bonnet et al. 2004) at the Very Large Telescope of ESO under variable conditions. SINFONI is an image slicer which operates between 1.1 and $2.4 \mu \mathrm{m}$. We used the seeing-limited mode with the largest available field of view of $8 " \times 8 "$ and a pixel scale of 250 mas. All data were taken with the $\mathrm{H}+\mathrm{K}$ grating which covers wavelengths between $1.45 \mu \mathrm{m}$ and $2.4 \mu \mathrm{m}$ at a spectral resolving power $\mathrm{R} \sim 1500(\sim 200 \mathrm{~km}$ $\mathrm{s}^{-1}$ ). We observed each galaxy for 180-230 min of on-source observing time, split into individual observations of 5 min length. Since most of our galaxies are smaller than the field of view, we adopted a dither pattern where the object is shifted between two opposite corners of the field of view. The spatial resolution of our data is limited by the size of the seeing disk, which is typically around $0.8 "$. They are measured from a standard star observed at the end of each hour of data taking. Data reduction procedures have been extensively explained in Nesvadba et al. $(2006,2008)$ and we do not describe them here.
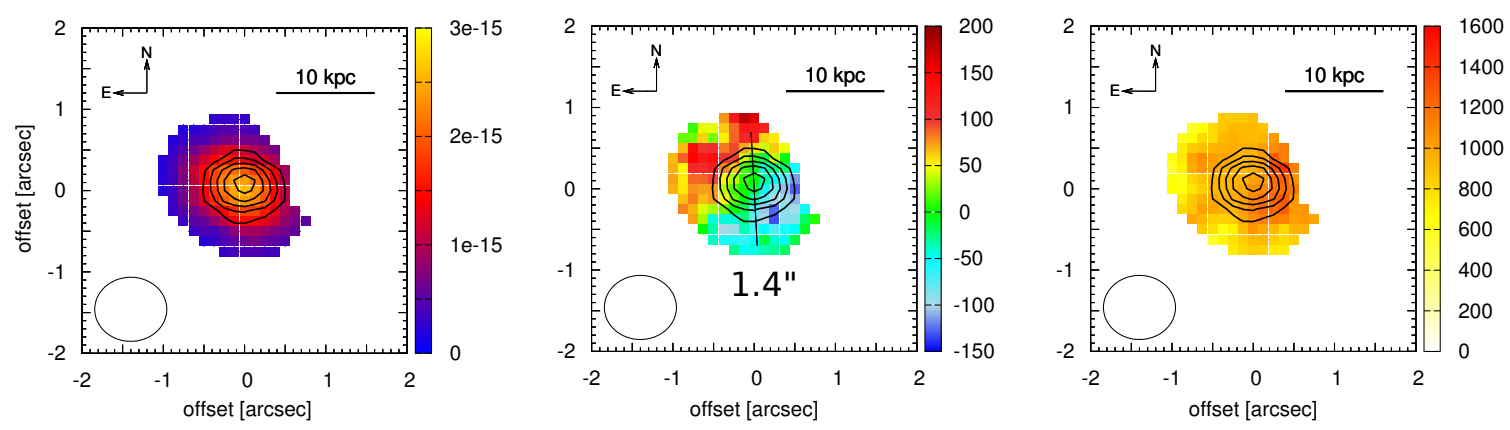

Figure 3: Surface brighthness (left, in $\mathrm{erg} \mathrm{s}^{-1} \mathrm{~cm}^{-2} \operatorname{arcsec}^{-2}$ ), velocity (center, in $\mathrm{km} \mathrm{s}^{-1}$ ) and velocity dispersion (right, in $\mathrm{km} \mathrm{s}^{-1}$ ) of NVSSJ012932-385433, one of the 9 moderate HzRG observed in our program. The seeing disk (FWHM) is shown in the lower left corner and the solid black line on the velocity map gives the size and the orientation of the radio jet. This galaxy illustrates how things become more ambiguous at lower radio powers: velocities are smaller (e.g., consistent with a rotating disk) meaning that gas may not escape the potential well of the galaxy, but in the same time velocity dispersions are as large as for the most powerful HzRGs. (see Collet et al. 2013, in prep. for details)

At moderate radio powers, we first note that sources are more diverse than at higher radio power: Line widths are generally high $\left(\sim 800 \mathrm{~km} \mathrm{~s}^{-1}\right)$ in regions where we detect radio emission, and more quiescent where we do not. These values, as large as for the most powerful sources, indicate that the jets affect the gas, but they do not always seem to produce very extended emissionline regions with high bulk velocities.

Hydrodynamical models (e.g., Wagner \& Bicknell 2011, 2012) show that jets permeate the gas overall, with a significant energy transfer, but it is not clear if the gas that escapes from the galaxy has high enough surface brightnesses to be easily seen, which could explain our observations. 
Although radio powers differ by 4 orders of magnitude, we can also compare this observation with results of Lehnert et al. (2011) who observed low bulk motions $\left(\sim 50 \mathrm{~km} \mathrm{~s}^{-1}\right)$ but high velocity dispersions $\left(\sim 500 \mathrm{~km} \mathrm{~s}^{-1}\right)$ through the Na D absorption line of 260 low-redshift radio galaxies.

Another difference with the most powerful HzRGs arethe lower masses of ionized gas observed in these objects, with $M_{\text {ion gas }} \sim 10^{8-9} M_{\odot}$. Whether these objects have less gas overall (i.e., they have similar ionized-to-molecular gas ratio $\frac{M_{i o n}}{M_{\text {mol }}} \sim 1$, similar to what is observed in the most powerful systems) or whether their gas content is closer to what is generally observed $\left(\frac{M_{\text {ion }}}{M_{\text {mol }}} \sim 10^{-2-3}\right)$ is still to be tested.

\section{Conclusion}

We observed 9 high-redshift radio galaxies with the VLT/SINFONI IFU in order to explore the impact of mechanical AGN feedback in systems with moderate radio power. Extended emission line regions of ionized gas with high velocity dispersions are the sign that the jets do perturbe the gas, but observed bulk velocities are not high enough to state that this gas will escape from the galaxy. Large quantities of ionized gas are observed in these systems $\left(M_{\text {ion gas }} \sim 10^{8-9} M_{\odot}\right)$ but this may not represent the majority of all their gas content. Complementary studies of other phases of the gas (e.g., molecular) are needed to obtain a global picture of the role of the radio jets in galaxy evolution.

\section{References}

[1] J. Silk \& M. Rees, 1998, Quasars and galaxy formation., A\&A 331 (L1-L4) [astro-ph/9801013]

[2] C.J. Willott et al., 2001, The radio luminosity function from the low-frequency 3CRR, 6CE and 7CRS complete samples., MNRAS 322 (536-552) [astro-ph/ 0010419$]$

[3] N.P.H. Nesvadba et al., 2006, Extreme gaz kinematics in the $z=2.2$ powerful radio galaxy MRC 1138-262: Evidence for efficient active galactic nucleus feedback in the early universe?, ApJ $\mathbf{6 5 0}$ (693-705) [astro-ph/0606530]

[4] D.J. Croton et al., 2006, The many lives of active galactic nuclei: Cooling flows, black holes and the luminosities and colours of galaxies., MNRAS 365 (11-28) [ast ro-ph/ 0508046 ]

[5] A. Sajina et al., 2007, Discovery of radio jets in $z \sim 2$ ultraluminous infrared galaxies with deep 9.7 $\mu m$ silicate absorption., ApJ 666 (L17-L20) [astro-ph/ 0708 . 0407]

[6] N.P.H. Nesvadba et al., 2008, Evidence for powerful active galactic nucleus winds at high redshift: Dynamics of galactic outflow in radio galaxies during the "Quasar Era"., A\&A 491 (407-424) [astro-ph/0809.5171]

[7] D.M. Alexander et al., 2010, Searching for evidence of energetic feedback in distant galaxies: a Galaxy wide outflow in a $z \simeq 2$ ultraluminous infrared galaxy., MNRAS 402 (2211-2220) [astro-ph/0911.0014]

[8] M.A. Gendre et al., 2010, The combined NVSS-FIRST galaxies (CoNFIG) sample - II. Comparison of space densities in the Fanaroff-Riley dichotomy, MNRAS 404 (1719-1732) [astro-ph/1001.4514]

[9] N.P.H. Nesvadba et al., 2011, The dynamics of the ionized and molecular interstellar medium in powerful obscured quasars at $z \geq 3.5$, () [astro-ph/1104.0937] 
[10] C.M. Harrison et al., 2012, Energetic galaxy-wide outflows in high-redshift ultraluminous infrared galaxies hosting AGN activity, MNRAS 426 (1073-1096) [astro-ph/1205. 1801]

[11] A. Sajina et al., 2008, Spitzer mid-infrared spectroscopy of infrared luminous galaxies at z $\sim 2$. III. Far-IR to radio properties and optical spectral diagnostics, ApJ 683 (659-682) [astro-ph/0805.0263]

[12] Eisenhauer et al., 2003, SINFONI - Integral field spectroscopy at 50 milli-arcsecond resolution with the ESO VLT., SPIE 4841 (1548-1561) [astro-ph/ 0306191 ]

[13] Bonnet et al., 2004, First light of SINFONI AO-module at VLT., SPIE 5490 (130-138)

[14] A.Y. Wagner \& G.V. Bicknell, 2011, Relativistic jet feedback in evolving galaxies, ApJ 728 (29-40) [astro-ph/1012.1092]

[15] A.Y. Wagner \& G.V. Bicknell, 2012, Driving outflows with relativistic jets and the dependence of active galactic nucleus feedback efficiency on interstellar medium inhomogeneity, ApJ 757 (136-160) [astro-ph/1205.0542]

[16] M.D. Lehnert et al., 2011, The Na D profiles of nearby low-power radio soures: jets powering outflows, A\&A 532 (L3-L8) [astro-ph/1107.1037] 